OPEN ACCESS

Edited by:

Shigeru Kamiya,

Kyorin University, Japan

Reviewed by:

Pinyi Lu,

Biotherapeutics, Inc., United States Julien Diana,

Institut National de la Santé et de la

Recherche Médicale, France

*Correspondence: Ikuo Kimura

ikimura@cc.tuat.ac.jp

Specialty section: This article was submitted to Nutritional Immunology, a section of the journal

Frontiers in Immunology

Received: 08 October 2017 Accepted: 11 December 2017 Published: 20 December 2017

Citation:

Li X, Watanabe K and Kimura I (2017) Gut Microbiota Dysbiosis Drives and Implies Novel Therapeutic Strategies for Diabetes Mellitus and Related Metabolic Diseases.

Front. Immunol. 8:1882. doi: 10.3389/fimmu.2017.01882

\section{Gut Microbiota Dysbiosis Drives and Implies Novel Therapeutic Strategies for Diabetes Mellitus and Related Metabolic Diseases}

\author{
Xuan $L^{1,2}{ }^{1,}$ Keita Watanabe ${ }^{1}$ and Ikuo Kimura ${ }^{1,2 *}$ \\ ${ }^{1}$ Department of Applied Biological Science, Graduate School of Agriculture, Tokyo University of Agriculture and Technology, \\ Fuchu-shi, Japan, ${ }^{2}$ AMED-CREST, Japan Agency for Medical Research and Development, Tokyo, Japan
}

Accumulating evidence over the past decade has linked the development of metabolic syndrome related to diabetes to variations in gut microbiota, an emerging, critical homeostatic regulator of host energy metabolism and immune responses. Mechanistic studies in rodent models have revealed an ever-increasing multitude of molecular mechanisms whereby the gut microbiota interacts with various host sensing and signaling pathways, leading to modulation of endocrine system, immune responses, nervous system activity, and hence, the predisposition to metabolic diseases. Remarkably, the microbiota-driven immune responses in metabolic tissues and the host nutrient-sensing mechanisms of gut microbial metabolites, in particular short-chain fatty acids, have been significantly associated with the proneness to diabetes and related disorders. This review will synthesize the recent efforts on unraveling the mediating role of gut microbiota in the pathogenesis of metabolic diseases, aiming to reveal new therapeutic opportunities.

\section{Keywords: gut microbiota dysbiosis, metabolic diseases, immune responses, nutrient-sensing receptors, energy metabolism \\ DIET-DRIVEN GUT MICROBIOTA DYSBIOSIS IS CAUSALLY LINKED TO THE TYPE II DIABETES MELLITUS (T2DM) AND RELATED METABOLIC DISORDERS}

Type II diabetes mellitus, a major constituent of metabolic diseases, is clinically hallmarked by hyperglycemia, impaired peripheral response to insulin (i.e., insulin resistance) and pancreatic $\beta$-cell decompensation. T2DM is essentially linked to obesity and the pathophysiology of both entails facets of gene-diet/lifestyle interactions. Obesity and T2DM also increase the incidence of non-alcoholic steatohepatitis and cardiovascular disease. Gut microbiota is a highly complex bacterial community that indigenously colonizes the gastrointestinal tract. It constitutes such a tremendous pool of microbial species and genetic variability that the alternations in its composition and repertoire of metabolites and components trigger markedly diverse host responses. To date, a wealth of evidence has emerged, among which a remarkable example is the replication of obese phenotypes of human discordant twin donors in rodent animals by fecal microbiota transplantation (1), substantiating the causative and/or mediatory role of gut microbiota particularly in the context of diet-induced metabolic diseases. 
The gut microbiota is dominated by the phyla Firmicutes and Bacteroidetes. Proteobacteria, Actinobacteria, Fusobacteria, Cyanobacteria, and Verrucomicrobia also occur but in much less abundance. The dysbiotic signatures in the gut microbiota associated with metabolic disease phenotypes include, remarkably, an increased ratio of Firmicutes to Bacteroidetes at phylum level $(2,3)$, an expansion of Proteobacteria $(4,5)$ and a reduced abundance of Akkermansia (6-9). Insulin-resistant phenotypes also exhibit a manifest proliferation of Prevotella copri and Bacteroides vulgatus, which elevate the circulating levels of branch chain amino acids (10). An obese microbiota was also found to associate with augmented serum glutamate levels due to the reduced abundance of Bacteroides thetaiotaomicron that converts glutamate (11). Moreover, an increased abundance of Proteobacteria and Escherichia coli with a reduction in the population of Firmicutes characterizes the gut microbiota associated with advanced fibrosis in human non-alcoholic fatty liver disease (NAFLD) (12). These dysbiotic microbiota configurations contribute to metabolic disorders by increasing energy harvest or by cross talking with the host immune, endocrine and nervous system via various nutrient sensing and signaling transduction mechanisms. Unraveling these mechanisms hence provides unique insights into the therapeutic opportunities for diabetes, obesity, and other metabolic diseases.

In light of the previous findings that have been efficiently summarized in several review articles (13-16), we would like to discuss the mechanistic basis of the emerging therapeutic strategies that target the host immune and nutrient-sensing pathways and to synthesize the most recent innovations and trends in the treatment of metabolic diseases.

\section{THE TRIALOGUE BETWEEN NUTRITIONAL STATUS, GUT MICROBIOTA, AND IMMUNE SYSTEM REVEALS NOVEL THERAPEUTIC OPPORTUNITIES FOR METABOLIC DISEASES}

Metabolic diseases are characterized by a state of chronic subclinical inflammation in metabolic tissues such as liver, adipose, muscles, and pancreatic islets. The causative role of a dysbiotic gut microbiota in this inflammatory status by virtue of engaging diverse signaling transduction pathways and immune responses has been increasingly established in the past decade. In light of the increasingly unraveled trialogue between diet, gut microbiota, and the host immune system, a multitude of therapeutic approaches against metabolic diseases have emerged. One compelling set of mechanisms dictate the translocation of commensal bacteria and bacterial fragments toward metabolic tissues, where they trigger pro-inflammatory responses at the early onset of metabolic disorders. Evidence suggests that this translocation is promoted by a diet/microbiota-driven gut barrier impairment in dysbiotic conditions, thereby continuously fueling the host immune machinery that orchestrates the innate and adaptive arms (Figure 1).
High-fat diet (HFD) is known to induce a lipopolysaccharide (LPS)-enriched intestinal microbiota with a consequently elevated plasma concentration of LPS, which characterizes a state of metabolic endotoxemia (17). In the intestinal tract, LPS triggers the dysfunction of the mucosal barrier. Specifically, HFD has been shown to induce an increase in gut permeability by impairing the expression of tight junction proteins (17) via a mechanism that involves LPS-induced activation of the toll-like receptor (TLR)-4 pathways (18), while prebiotic carbohydrates treatment help reverse this impairment via a microbiota-driven modulation of endogenous proglucagon-derived peptide (GLP-2) by enteroendocrine L cells (19). HFD also reduces the mucus layer thickness, which however could be normalized upon administration of the mucin-producing Akkermansia muciniphila or prebiotics that restore its abundance via the endocannabinoids system that essentially regulate the gut barrier function (6). HFD has also been shown to cause a loss of intestinal T helper (Th) 17 cells putatively through an altered gut microbiota that interfere with the induction of Th17 cells by the antigen-presenting cells, resulting in diminished intestinal defense and integrity (20). Adoptive transfer of Th17 cells to obese mice shapes the gut microbiota to resemble that of a lean phenotype by increasing the ratio of Bacteroidetes to Firmicutes and the abundance of Akkermansia, contributing to improved metabolic profile to the recipients (21). Not surprisingly, HFD also impairs the induction of interleukin (IL)-22, which plays an essential role in eliciting antimicrobial immunity and maintaining mucosal barrier integrity within the intestine. Accordingly, promoting the production of IL-22 can be a potential intervention strategy to restore mucosal barrier integrity to reduce tissue inflammation and to improve metabolic profile (22). Moreover, it has been demonstrated that immunization with the HFD-associated ileum microbial extracts reversed partially gut microbiota dysbiosis and prevented hyperglycemia and insulin resistance in response to HFD likely by enhancing the proliferation of intestinal CD4 T cells (23). This underlines the potential of vaccination for preventing and managing T2DM.

The mechanism of bacterial translocation involves the host recognition receptors for the microbe-associated molecular patterns, including CD14 that binds bacterial LPS and the peptidoglycan (PG) sensor NOD1 (24). Of note, sensing of PG by NOD2 confers, however, protection in HFD-induced metabolic disease against gut barrier impairments and the microbiotadriven tissue inflammation $(25,26)$. It follows that manipulation of intestinal bacterial adherence, bacterial translocation and receptors for bacterial fragments could be promising strategies to prevent or revert the incidence of metabolic disorders. For example, treatments with probiotics, such as Bifidobacteria $(24,27)$ and Akkermansia (28), have been demonstrated to be efficacious against the adherence and translocation of mucosal bacteria, tissue inflammation, and insulin resistance by modulating the gut microbial structure. The administration of polyphenols as prebiotics also confers similar protective effects in diet-induced metabolic syndrome by increasing the abundance of Akkermansia (29). The role of bacterial muramyl dipeptide as a postbiotic has also been documented in reducing inflammation and promoting insulin signaling in the state of metabolic endotoxemia, glycemia, and obesity via a pathway that involves NOD2 (30). 


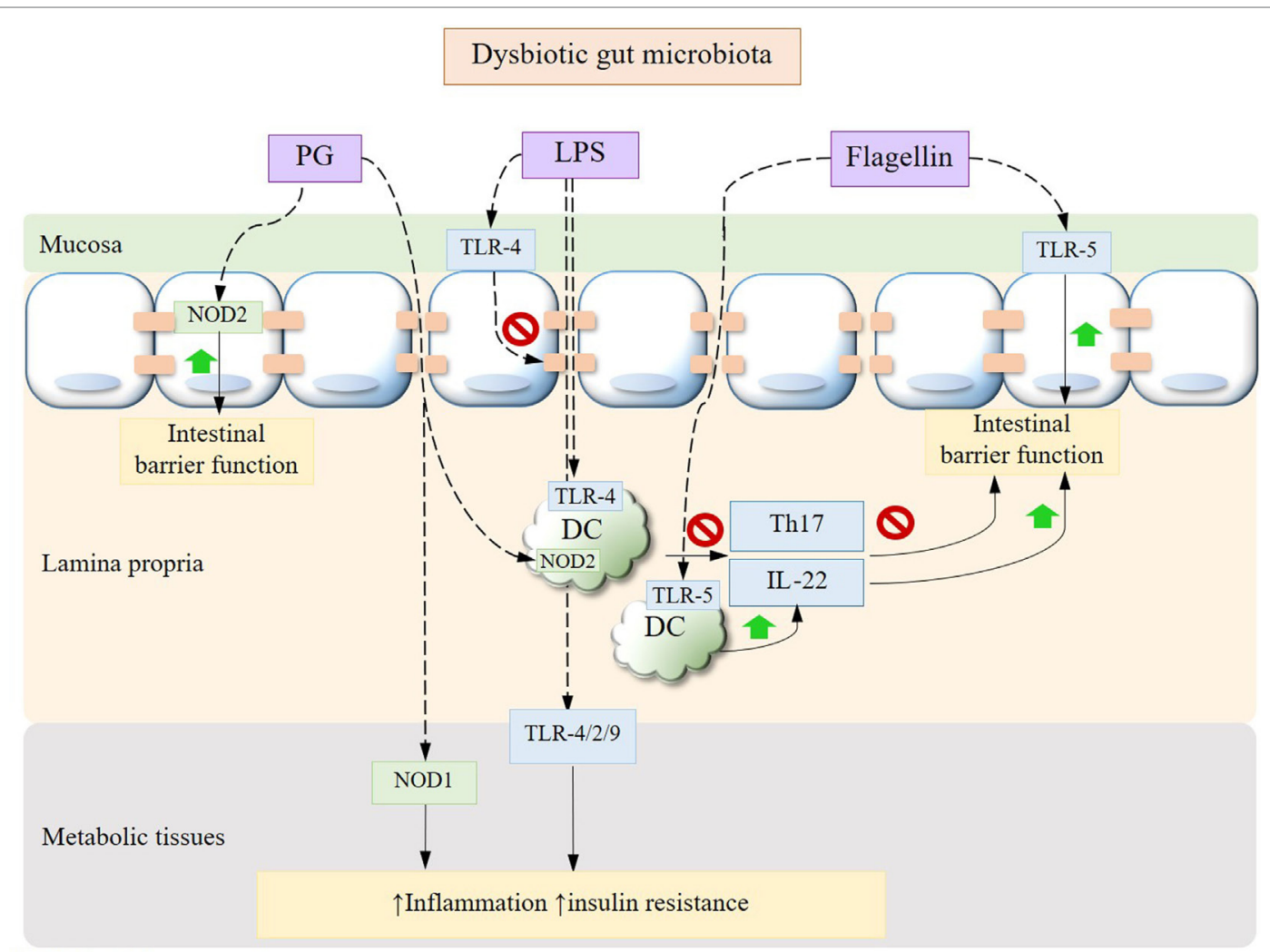

FIGURE 1 | Gut microbiota dysbiosis-driven immune signaling pathways. Bacterial translocation occurs secondary to the mucosal epithelial barrier impairment driven by dysbiotic alterations in gut microbiota, leading to elevated circulating and tissue MAMPs such as LPS and PG. Bacterial LPS can disrupt the expression of epithelial tight junctions and, upon being translocated to peripheral tissues, trigger inflammation, and insulin resistance through toll-like receptors (TLRs). While PG induces tissue inflammation via NOD1, its recognition by NOD2 in intestinal epithelium confers protection against gut barrier dysfunction. The cross talk between APC and Th17 cells is also impaired under dysbiotic conditions with a decrease in IL-22. Adversely, the interactions between bacterial flagellin and intestinal epithelium or APC via TLR-5 improve gut barrier function. MAMPs, microbe-associated molecular patterns; LPS, lipopolysaccharide; PG, peptidoglycan; APC, antigen-presenting cells; IL, interleukin.

Upon being translocated to metabolic tissues, LPS can elicit pro-inflammatory responses via pathways mediated primarily by TLR-4 (31) with the involvement of TLR-2 (32) and TLR-9 (33), diminishing insulin signaling and increasing adiposity in the state of obesity and T2DM. This cross talk is mediated by gut microbiota as shown by the abrogation of these metabolic endotoxemia-induced phenotypes along with a decrease in inflammation markers and intestinal permeability after an antibiotic treatment (17). Anti-inflammatory agents, such as 5 -aminosalicylic acid, therefore prove to be efficacious in restoring gut barrier and reducing tissue microbiota dysbiosis and inflammation in metabolic syndrome (34). The attenuation in metabolic endotoxemia also serves as one mechanism that contributes to the resolution of insulin resistance and T2DM after Roux-en-Y gastric bypass (RYGB) (35). The RYGB-associated, markedly diminished endogenous (i.e., microbiota derived), LPS highlights the role of a modulated gut microbiota as a mediator of metabolic conditions (36). Gut microbial homeostasis is also subject to regulation by intestinal inflammasomes, the loss of which can induce a dysbiotic microbiome and exacerbate metabolic endotoxemia, as observed in the liver, leading to the development of hallmark features of NAFLD including increased hepatic steatosis, inflammation, and insulin resistance (33). Metabolic endotoxemia also induces endoplasmic reticulum stress and concomitantly the activity of histone acetyltransferase p300, which acetylates insulin receptor substrate, prevents its association with the insulin receptor and hence impairs insulin signaling (37). Likewise, TLR-5-dependent signaling regulates the gut microbial ecology by sensing bacterial flagellin, conferring protection against metabolic diseases $(38,39)$. Myeloid differentiation primary response $88(\mathrm{MyD} 88)$ is an important signaling component of TLR pathways. It is worth noting that tissue-specific MyD88 deficiency confers distinct phenotypes. Specifically, intestinal specific deletion of MyD88 putatively serves to normalize the gut barrier function and increase energy expenditure during HFD feeding in a microbiota-dependent manner, leading to reduced adiposity and inflammation and an improved glucose homeostasis (40). Conversely, hepatocyte-specific deletion of MyD88 disrupts glucose and lipid homeostasis presumably by inducing changes in the related gene expression, bile acid signaling, and in the gut microbiota configuration (41). Deletion of MyD88 has also been demonstrated to reduce the 


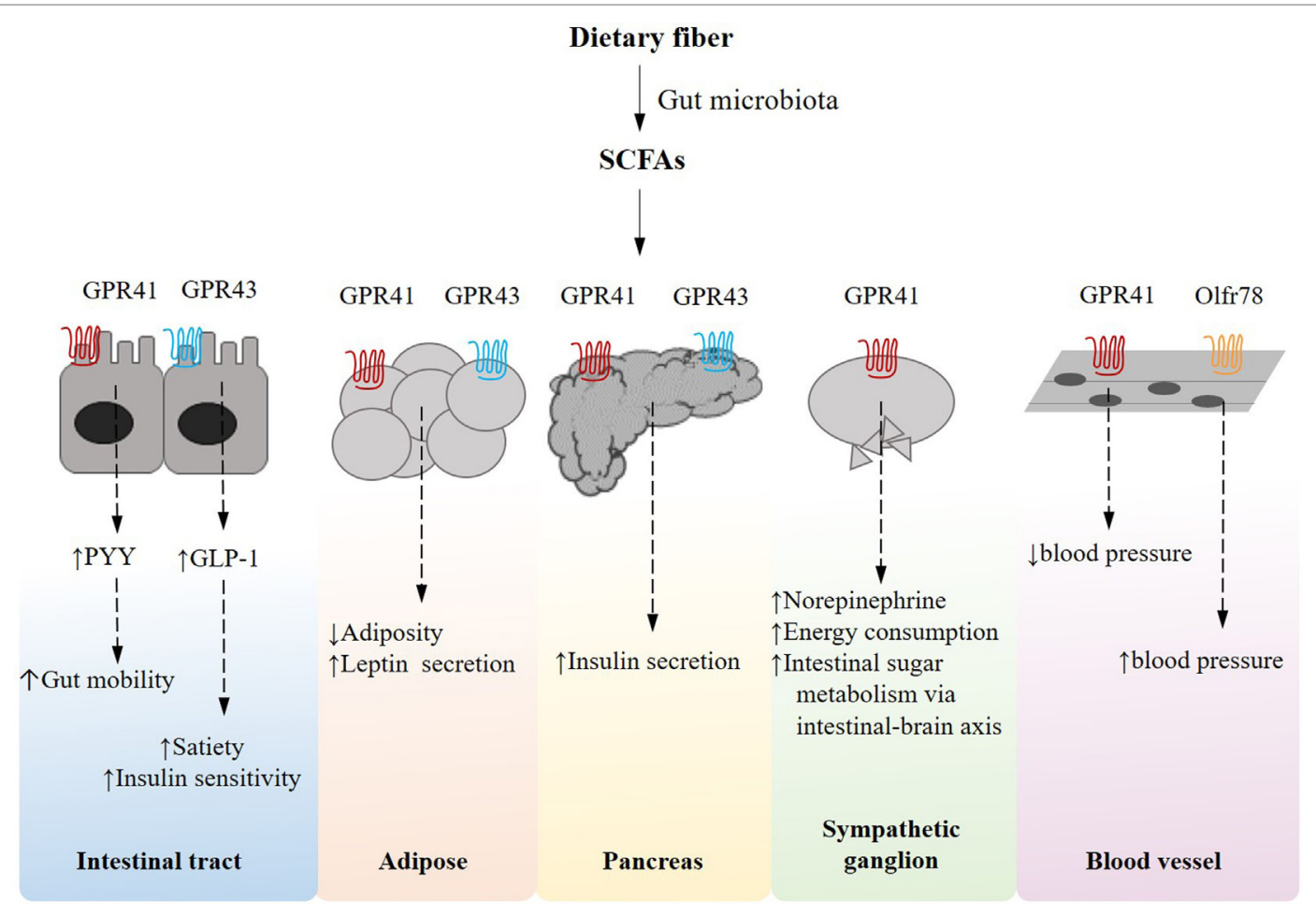

FIGURE 2 | Short-chain fatty acid (SCFA)-receptor-mediated pathways and their effects on host energy metabolism in peripheral tissues. Gut microbes can ferment dietary fiber into SCFAs, which induce an array of G-protein coupled receptor-mediated signaling pathways that are essentially implicated in host energy homeostasis in multiple tissues.

incidence of spontaneous type I diabetes by inducing transmissible changes in the gut microbiota (42). These findings support the role of MyD88 as a pharmacological target for metabolic disease, though it multifaceted functions warrant further investigation.

\section{NUTRIENT-SENSING MECHANISMS ARE KEY METABOLIC MEDIATORS OF HOST RESPONSES TO GUT MICROBIOTA ALTERATIONS}

It has been increasingly established that in the metabolic machinery G-protein coupled receptor (GPCR)-mediated nutrient sensing serves as essential mechanisms that coordinate host responses to dietary and endogenous nutrients, gaining significant interest as a putative therapeutic target for metabolic diseases. A distinct subset of the nutrient-sensing receptors is specifically activated by free fatty acids (FFAs) of various chain lengths. In particular, short-chain fatty acids (SCFAs), including primarily acetate, propionate, and butyrate, are the most abundant microbial metabolites derived from the otherwise indigestible dietary polysaccharides and the natural ligands for fatty acid receptors such as GPR41/FFAR3, GPR43/FFAR2, GPR109A, and Olfr78 (Figure 2). Activation of the GPR41 expressed on the enteroendocrine cells is reported to stimulate the secretion of the gut hormone peptide YY, which functions to reduce energy intake $(43,44)$, while sensing of SCFAs by GPR43 triggers the production of glucagon-like peptide-1 (GLP-1), which is known to decrease gastric emptying rate and improve glucose-induced insulin secretion (44-46). GPR41 and GPR43 also occur on intestinal epithelial cells, upon stimulation by SCFAs, playing protective role against intestinal inflammation by inducing inflammasome activation (47). A propionate-dependent signaling of GPR41 is also involved in a gut-brain neural circuit that regulates intestinal gluconeogenesis, a substantial mechanism that ensures metabolic homeostasis (48). In adipose tissues, SCFA-GPR41 interaction correlates with the circulating level of leptin, thereby regulating food intake (49), while GPR43 mediates insulin signaling and adipogenesis (50). SCFAs, acting through GPR41 expressed in neurons of the superior cervical ganglion, also contribute to increased energy expenditure by increasing sympathetic nervous system activity (51). SCFAs, in addition, have been suggested to regulate the proliferation of pancreatic $\beta$ cells and insulin biosynthesis via GPR41 or GPR43 (52-55). GPR109A is a receptor for niacin but also responds to butyrate, contributing to reduced colonic inflammation and homeostatic lipid metabolism in adipose tissues (56). On the other hand, SCFAs play a role in blood pressure regulation via the receptors in blood vessels. Specifically, the activation of GPR41 has been observed to reduce blood pressure (57), whereas an olfactory receptor Olfr78, upon stimulation by acetate and propionate, leads to an increased blood pressure (58). It is also worth noting that endogenously derived SCFAs confer an epigenetic mechanism whereby chromatin structure and cell fate allocation respond to 
TABLE 1 | Potential therapeutic targets and strategies revealed by the mechanistic basis of the pathophysiology of metabolic diseases.

\begin{tabular}{lc}
\hline Potential therapeutic target and strategy & Reference \\
\hline Prebiotics (e.g., carbohydrates and polyphenols) & $(6,19,29)$ \\
Probiotics (e.g., Akkermansia muciniphila and Bifidobacteria) & $(6,24,27,28)$ \\
Postbiotics (e.g., bacterial muramyl dipeptide) & $(30)$ \\
Adoptive transfer of T helper 17 cells & $(20)$ \\
Anti-inflammatory agents (e.g., 5-aminosalicylic acid) & $(34)$ \\
Immunization with the high-fat diet-associated ileum microbial & $(23)$ \\
extracts & \\
NOD1 antagonists & $(24)$ \\
NOD2 agonists & $(25,26)$ \\
Myd88 agonists or antagonists & $(40-42)$ \\
Toll-like receptor-5 agonists & $(38,39)$ \\
Improving gut microbial production of short-chain fatty acids & $(21,59,60)$ \\
Farnesoid X receptor antagonists & $(67,68)$ \\
GPR41 agonists & $(44,47,48,51)$ \\
GPR43 agonists & $(44,45,50)$ \\
GPR109A agonists & $(56)$ \\
Olfr78 antagonists & $(58)$ \\
GPR119 agonists & $(63-65)$ \\
Genetic modulation of microbiota-derived metabolites & $(66)$ \\
\hline
\end{tabular}

gut microbiota and diet $(21,59,60)$. Taken together, it follows that nutrient-sensing receptors could be therapeutic targets and their agonism a potent strategy that enhances insulin signaling, energy homeostasis and intestinal function, highlighting the significance of dietary microbiota-accessible carbohydrates (61) and the development of new agonists for the treatment of metabolic diseases. Despite the evidence supportive of the beneficial role of dietary SCFAs in maintaining metabolic homeostasis, controversially, for instance, a microbiota-dependent excessive accumulation of SCFAs driven by immunodeficiency of TLR-5 signaling has been documented to promote low-grade inflammation, augmenting hepatic de novo lipogenesis and insulin resistance (62). This discrepancy warrants further studies on the role of SCFAs and their receptors in the context of disease model, dose, and tissue dependence.

In addition to the SCFA-dependent GPCRs, GPR119 recognizes long-chain fatty acids and derivatives as endogenous ligands. It is worth mentioning that GPR119 is a validated therapeutic target

\section{REFERENCES}

1. Ridaura V, Faith J, Rey F, Cheng J, Duncan A, Kau A, et al. Gut microbiota from twins discordant for obesity modulate metabolism in mice. Science (2013) 341:1241214. doi:10.1126/science.1241214

2. Ley RE, Turnbaugh PJ, Klein S, Gordan JI. Microbial ecology: human gut microbes associated with obesity. Nature (2006) 444:1022-3. doi:10.1038/ 4441022a

3. Turnbaugh PJ, Ley RE, Mahowald MA, Magrini V, Mardis ER, Gordan JI. An obesity-associated gut microbiome with increased capacity for energy harvest. Nature (2006) 444:1027-31. doi:10.1038/nature05414

4. Martinez-Medina M, Denizot J, Dreux N, Robin F, Billard E, Bonnet R, et al. Western diet induces dysbiosis with increased E. coli in CEABAC10 mice, alters host barrier function favouring AIEC colonisation. Gut (2014) 63:116-24. doi:10.1136/gutjnl-2012-304119

5. Raso GM, Simeoli R, Iacono A, Santoro A, Amero P, Paciello O, et al. Effects of a Lactobacillus paracasei B21060 based synbiotic on steatosis, insulin signaling for T2DM in that it essentially mediates glucose-induced insulin secretion and intestinal secretion of GLP-1 and glucose-dependent insulinotropic peptide (63-65). Recently, Cohen et al. (66) identified striking structural and functional similarities between human ligands for GPR119 and their microbiota-encoded counterparts, proposing a new perspective on antidiabetic agents that dictates genetic modulation of microbiota-derived metabolites to elicit desirable host responses.

Nuclear farnesoid X receptor (FXR)-mediated bile acid signaling also represents a nutrient-sensing mechanism that regulates glucose and lipid metabolism. Gut microbiota plays a pivotal role in the biotransformation and thereby the signaling of bile acids. It has been recently reported that the obese phenotype is associated with a gut microbiota that promotes deconjugation of bile acids, resulting in increased hepatic steatosis and weight gain via the activation of FXR $(67,68)$, which may bear implications for treating metabolic diseases.

\section{CONCLUDING REMARKS}

As the dysbiosis of gut microbiota is increasingly appreciated as a mechanism accounting for metabolic diseases, the growing understanding of microbiota-driven immune and metabolic responses has begun to reveal potential interventional targets, sustaining the development of new therapeutic strategies by dietary and/or pharmacological means. These progresses highlight the therapeutic significance of (1) dietary intervention (e.g., prebiotics, probiotics, and postbiotics) and fecal microbiota transplantation that tackle microbiota dysbiosis and (2) manipulation of the key players in the intestinal integrity and function, bacterial recognition and translocation, tissue inflammation, and host nutrient-sensing mechanisms by using such as receptor agonists/antagonists and anti-inflammatory agents; in particular, recent reports about vaccination and genetic modulation of the gut microbial metabolites further add to our knowledge of the therapeutics of metabolic diseases (Table 1).

\section{AUTHOR CONTRIBUTIONS}

$\mathrm{XL}$ and KW wrote the paper. IK supervised and wrote the paper. and toll-like receptor expression in rats fed a high-fat diet. J Nutr Biochem (2014) 25:81-90. doi:10.1016/j.jnutbio.2013.09.006

6. Everard A, Belzer C, Geurts L, Ouwerkerk J, Druart C, Bindels L, et al. Crosstalk between Akkermansia muciniphila and intestinal epithelium controls diet-induced obesity. Proc Natl Acad Sci U S A (2013) 110:9066-71. doi:10.1073/ pnas. 1219451110

7. Schneeberger M, Everard A, Gómez-Valadés AG, Matamoros S, Ramírez S, Delzenne NM, et al. Akkermansia muciniphila inversely correlates with the onset of inflammation, altered adipose tissue metabolism and metabolic disorders during obesity in mice. Sci Rep (2015) 5:16643. doi:10.1038/srep 16643

8. Greer RL, Dong X, Moraes ACF, Zielke RA, Fernandes GR, Peremyslova E, et al. Akkermansia muciniphila mediates negative effects of IFN $\gamma$ on glucose metabolism. Nat Commun (2016) 7:13329. doi:10.1038/ncomms13329

9. Plovier H, Everard A, Druart C, Depommier C, Van Hul M, Geurts L, et al. A purified membrane protein from Akkermansia muciniphila or the pasteurized bacterium improves metabolism in obese and diabetic mice. Nat Med (2017) 23:107-13. doi:10.1038/nm.4236 
10. Pedersen HK, Gudmundsdottir V, Nielsen HB, Hyotylainen T, Nielsen T, Jensen BA, et al. Human gut microbes impact host serum metabolome and insulin sensitivity. Nature (2016) 535:376-81. doi:10.1038/nature18646

11. Liu R, Hong J, Xu X, Feng Q, Zhang D, Gu Y, et al. Gut microbiome and serum metabolome alterations in obesity and after weight-loss intervention. Nat Med (2017) 23:859-68. doi:10.1038/nm.4358

12. Loomba R, Seguritan V, Li W, Long T, Klitgord N, Bhatt A, et al. Gut microbiome-based metagenomic signature for non-invasive detection of advanced fibrosis in human nonalcoholic fatty liver disease. Cell Metab (2017) 25:1054-62.e5. doi:10.1016/j.cmet.2017.04.001

13. Sanz Y, Olivares M, Moya-Pérez Á, Agostoni C. Understanding the role of gut microbiome in metabolic disease risk. Pediatr Res (2014) 77:236-44. doi:10.1038/pr.2014.170

14. Hur K, Lee M. Gut microbiota and metabolic disorders. Diabetes Metab J (2015) 39:198. doi:10.4093/dmj.2015.39.3.198

15. Boulangé C, Neves A, Chilloux J, Nicholson J, Dumas M. Impact of the gut microbiota on inflammation, obesity, and metabolic disease. Genome Med (2016) 8:42. doi:10.1186/s13073-016-0303-2

16. Yang J, Kweon M. The gut microbiota: a key regulator of metabolic diseases. BMB Rep (2016) 49:536-41. doi:10.5483/bmbrep.2016.49.10.144

17. Cani P, Bibiloni R, Knauf C, Waget A, Neyrinck A, Delzenne N, et al. Changes in gut microbiota control metabolic endotoxemia-induced inflammation in high-fat diet-induced obesity and diabetes in mice. Diabetes (2008) 57:1470-81. doi:10.2337/db07-1403

18. Guo S, Nighot M, Al-Sadi R, Alhmoud T, Nighot P, Ma T. Lipopolysaccharide regulation of intestinal tight junction permeability is mediated by TLR4 signal transduction pathway activation of FAK and MyD88. J Immunol (2015) 195:4999-5010. doi:10.4049/jimmunol.1402598

19. Cani P, Possemiers S, Van de Wiele T, Guiot Y, Everard A, Rottier O, et al. Changes in gut microbiota control inflammation in obese mice through a mechanism involving GLP-2-driven improvement of gut permeability. Gut (2009) 58:1091-103. doi:10.1136/gut.2008.165886

20. Garidou L, Pomié C, Klopp P, Waget A, Charpentier J, Aloulou M, et al. The gut microbiota regulates intestinal CD4 T cells expressing ROR $\gamma \mathrm{t}$ and controls metabolic disease. Cell Metab (2015) 22:100-12. doi:10.1016/j. cmet.2015.06.001

21. Hong C, Park A, Yang B, Yun C, Kwak M, Lee G, et al. Gut-specific delivery of T-helper 17 cells reduces obesity and insulin resistance in mice. Gastroenterology (2017) 152:1998-2010. doi:10.1053/j.gastro.2017.02.016

22. Wang X, Ota N, Manzanillo P, Kates L, Zavala-Solorio J, Eidenschenk C, et al. Interleukin-22 alleviates metabolic disorders and restores mucosal immunity in diabetes. Nature (2014) 514:237-41. doi:10.1038/nature13564

23. Pomié $\mathrm{C}$, Blasco-Baque $\mathrm{V}$, Klopp $\mathrm{P}$, Nicolas $\mathrm{S}$, Waget $\mathrm{A}$, Loubières $\mathrm{P}$, et al. Triggering the adaptive immune system with commensal gut bacteria protects against insulin resistance and dysglycemia. Mol Metab (2016) 5:392-403. doi:10.1016/j.molmet.2016.03.004

24. Amar J, Chabo C, Waget A, Klopp P, Vachoux C, Bermúdez-Humarán L, et al. Intestinal mucosal adherence and translocation of commensal bacteria at the early onset of type 2 diabetes: molecular mechanisms and probiotic treatment. EMBO Mol Med (2011) 3(9):559-72. doi:10.1002/emmm. 201100159

25. Barreau F, Madre C, Meinzer U, Berrebi D, Dussaillant M, Merlin F, et al. Nod2 regulates the host response towards microflora by modulating $\mathrm{T}$ cell function and epithelial permeability in mouse Peyer's patches. Gut (2010) 59:207-17. doi:10.1136/gut.2008.171546

26. Denou E, Lolmede K, Garidou L, Pomie C, Chabo C, Lau T, et al. Defective NOD2 peptidoglycan sensing promotes diet-induced inflammation, dysbiosis, and insulin resistance. EMBO Mol Med (2015) 7:259-74. doi:10.15252/ emmm.201404169

27. Moya-Pérez A, Neef A, Sanz Y. Bifidobacterium pseudocatenulatum CECT 7765 reduces obesity-associated inflammation by restoring the lymphocyte-macrophage balance and gut microbiota structure in high-fat diet-fed mice. PLoS One (2015) 10:e0126976. doi:10.1371/journal.pone.0126976

28. Shin N, Lee J, Lee H, Kim M, Whon T, Lee M, et al. An increase in the Akkermansia spp. population induced by metformin treatment improves glucose homeostasis in diet-induced obese mice. Gut (2014) 63:727-35. doi:10.1136/gutjnl-2012-303839

29. Anhê F, Roy D, Pilon G, Dudonné S, Matamoros S, Varin T, et al. A polyphenol-rich cranberry extract protects from diet-induced obesity, insulin resistance and intestinal inflammation in association with increased Akkermansia spp. population in the gut microbiota of mice. Gut (2014) 64:872-83. doi:10.1136/gutjnl-2014-307142

30. Cavallari J, Fullerton M, Duggan B, Foley K, Denou E, Smith B, et al. Muramyl dipeptide-based postbiotics mitigate obesity-induced insulin resistance via IRF4. Cell Metab (2017) 25:1063-74.e3. doi:10.1016/j.cmet.2017.03.021

31. Cani P, Amar J, Iglesias M, Poggi M, Knauf C, Bastelica D, et al. Metabolic endotoxemia initiates obesity and insulin resistance. Diabetes (2007) 56:1761-72. doi:10.2337/db06-1491

32. Creely S, McTernan P, Kusminski C, Fisher F, Da Silva N, Khanolkar M, et al. Lipopolysaccharide activates an innate immune system response in human adipose tissue in obesity and type 2 diabetes. Am J Physiol Endocrinol Metab (2007) 292:E740-7. doi:10.1152/ajpendo.00302.2006

33. Henao-Mejia J, Elinav E, Jin C, Hao L, Mehal W, Strowig T, et al. Inflammasomemediated dysbiosis regulates progression of NAFLD and obesity. Nature (2012) 482:179-85. doi:10.1038/nature10809

34. Luck H, Tsai S, Chung J, Clemente-Casares X, Ghazarian M, Revelo X, et al. Regulation of obesity-related insulin resistance with gut anti-inflammatory agents. Cell Metab (2015) 21:527-42. doi:10.1016/j.cmet.2015.03.001

35. Monte S, Caruana J, Ghanim H, Sia C, Korzeniewski K, Schentag J, et al. Reduction in endotoxemia, oxidative and inflammatory stress, and insulin resistance after Roux-en-Y gastric bypass surgery in patients with morbid obesity and type 2 diabetes mellitus. Surgery (2012) 151:587-93. doi:10.1016/j. surg.2011.09.038

36. Luche E, Cousin B, Garidou L, Serino M, Waget A, Barreau C, et al. Metabolic endotoxemia directly increases the proliferation of adipocyte precursors at the onset of metabolic diseases through a CD14-dependent mechanism. Mol Metab (2013) 2:281-91. doi:10.1016/j.molmet.2013.06.005

37. Cao J, Peng J, An H, He Q, Boronina T, Guo S, et al. Endotoxemia-mediated activation of acetyltransferase P300 impairs insulin signaling in obesity. Nat Commun (2017) 8:131. doi:10.1038/s41467-017-00163-w

38. Vijay-Kumar M, Aitken J, Carvalho F, Cullender T, Mwangi S, Srinivasan S, et al. Metabolic syndrome and altered gut microbiota in mice lacking toll-like receptor 5. Science (2010) 328:228-31. doi:10.1126/science.1179721

39. Chassaing B, Ley R, Gewirtz A. Intestinal epithelial cell toll-like receptor 5 regulates the intestinal microbiota to prevent low-grade inflammation and metabolic syndrome in mice. Gastroenterology (2014) 147:1363-77.e17. doi:10.1053/j.gastro.2014.08.033

40. Everard A, Geurts L, Caesar R, Van Hul M, Matamoros S, Duparc T, et al. Intestinal epithelial MyD88 is a sensor switching host metabolism towards obesity according to nutritional status. Nat Commun (2014) 5:5648. doi: $10.1038 /$ ncomms6648

41. Duparc T, Plovier H, Marrachelli V, Van Hul M, Essaghir A, Ståhlman M, et al. Hepatocyte MyD88 affects bile acids, gut microbiota and metabolome contributing to regulate glucose and lipid metabolism. Gut (2017) 66:620-32. doi:10.1136/gutjnl-2015-310904

42. Wen L, Ley R, Volchkov P, Stranges P, Avanesyan L, Stonebraker A, et al. Innate immunity and intestinal microbiota in the development of type 1 diabetes. Nature (2008) 455:1109-13. doi:10.1038/nature07336

43. Samuel BS, Shaito A, Motoike T, Rey F, Backhed F, Manchester JK, et al. Effects of the gut microbiota on host adiposity are modulated by the short-chain fatty-acid binding G protein-coupled receptor, Gpr41. Proc Natl Acad Sci U S A (2008) 105:16767-72. doi:10.1073/pnas.0808567105

44. Chambers ES, Viardot A, Psichas A, Morrison DJ, Murphy KG, Zac-Varghese SE, et al. Effects of targeted delivery of propionate to the human colon on appetite regulation, body weight maintenance and adiposity in overweight adults. Gut (2015) 64:1744-54. doi:10.1136/gutjnl-2014-307913

45. Tolhurst G, Heffron H, Lam YS, Parker HE, Habib AM, Diakogiannaki E, et al. Short-chain fatty acids stimulate glucagon-like peptide-1 secretion via the G-protein-coupled receptor FFAR2. Diabetes (2012) 61:364-71. doi:10.2337/ db11-1019

46. Grasset E, Puel A, Charpentier J, Collet X, Christensen J, Tercé F, et al. A specific gut microbiota dysbiosis of type 2 diabetic mice induces GLP-1 resistance through an enteric NO-dependent and gut-brain axis mechanism. Cell Metab (2017) 25:1075-90.e5. doi:10.1016/j.cmet.2017.04.013

47. Macia L, Tan J, Vieira A, Leach K, Stanley D, Luong S, et al. Metabolite-sensing receptors GPR43 and GPR109A facilitate dietary fibre-induced gut homeostasis through regulation of the inflammasome. Nat Commun (2015) 6:6734. doi:10.1038/ncomms7734 
48. De Vadder F, Kovatcheva-Datchary P, Goncalves D, Vinera J, Zitoun C, Duchampt A, et al. Microbiota-generated metabolites promote metabolic benefits via gut-brain neural circuits. Cell (2014) 156:84-96. doi:10.1016/j. cell.2013.12.016

49. Zaibi MS, Stocker CJ, O’Dowd J, Davies A, Bellahcene M, Cawthorne MA, et al. Roles of GPR41 and GPR43 in leptin secretory responses of murine adipocytes to short chain fatty acids. FEBS Lett (2010) 584:2381-6. doi:10.1016/j. febslet.2010.04.027

50. Kimura I, Ozawa K, Inoue D, Imamura T, Kimura K, Maeda T, et al. The gut microbiota suppresses insulin-mediated fat accumulation via the shortchain fatty acid receptor GPR43. Nat Commun (2013) 4:1829. doi:10.1038/ ncomms 2852

51. Kimura I, Inoue D, Maeda T, Hara T, Ichimura A, Miyauchi S, et al. Shortchain fatty acids and ketones directly regulate sympathetic nervous system via G protein-coupled receptor 41 (GPR41). Proc Natl Acad Sci U S A (2011) 108:8030-5. doi:10.1073/pnas.1016088108

52. McNelis JC, Lee YS, Mayoral R, van der Kant R, Johnson AMF, Wollam J, et al. GPR43 potentiates $\beta$-cell function in obesity. Diabetes (2015) 64:3203-17. doi:10.2337/db14-1938

53. Priyadarshini M, Villa S, Fuller M, Wicksteed B, Mackay C, Alquier T, et al. An acetate-specific GPCR, FFAR2, regulates insulin secretion. Mol Endocrinol (2015) 29:1055-66. doi:10.1210/me.2015-1007

54. Tang C, Ahmed K, Gille A, Lu S, Gröne HJ, Tunaru S, et al. Loss of FFA2 and FFA3 increases insulin secretion and improves glucose tolerance in type 2 diabetes. Nat Med (2015) 21:173-7. doi:10.1038/nm.3779

55. Veprik A, Laufer D, Weiss S, Rubins N, Walker M. GPR41 modulates insulin secretion and gene expression in pancreatic-cells and modifies metabolic homeostasis in fed and fasting states. FASEB J (2016) 30:3860-9. doi:10.1096/ f. $201500030 \mathrm{R}$

56. Singh N, Gurav A, Sivaprakasam S, Brady E, Padia R, Shi H, et al. Activation of Gpr109a, receptor for niacin and the commensal metabolite butyrate, suppresses colonic inflammation and carcinogenesis. Immunity (2014) 40:128-39. doi:10.1016/j.immuni.2013.12.007

57. Natarajan N, Hori D, Flavahan S, Steppan J, Flavahan N, Berkowitz D, et al. Microbial short chain fatty acid metabolites lower blood pressure via endothelial G protein-coupled receptor 41. Physiol Genomics (2016) 48:826-34. doi:10.1152/physiolgenomics.00089.2016

58. Pluznick JL, Protzko RJ, Gevorgyan H, Peterlin Z, Sipos A, Han J, et al. Olfactory receptor responding to gut microbiota-derived signals plays a role in renin secretion and blood pressure regulation. Proc Natl Acad Sci U S A (2013) 110:4410-5. doi:10.1073/pnas.1215927110

59. Furusawa Y, Obata Y, Fukuda S, Endo TA, Nakato G, Takahashi D, et al. Commensal microbe-derived butyrate induces the differentiation of colonic regulatory T cells. Nature (2013) 504:446-50. doi:10.1038/nature12721
60. Remely M, Aumueller E, Merold C, Dworzak S, Hippe B, Zanner J, et al. Effects of short chain fatty acid producing bacteria on epigenetic regulation of FFAR3 in type 2 diabetes and obesity. Gene (2014) 537:85-92. doi:10.1016/j. gene.2013.11.081

61. Sonnenburg E, Smits S, Tikhonov M, Higginbottom S, Wingreen N, Sonnenburg J. Diet-induced extinctions in the gut microbiota compound over generations. Nature (2016) 529:212-5. doi:10.1038/nature16504

62. Singh V, Chassaing B, Zhang L, San Yeoh B, Xiao X, Kumar M, et al. Microbiotadependent hepatic lipogenesis mediated by stearoyl CoA desaturase 1 (SCD1) promotes metabolic syndrome in TLR5-deficient mice. Cell Metab (2015) 22:983-96. doi:10.1016/j.cmet.2015.09.028

63. Katz L, Gambale J, Rothenberg P, Vanapalli S, Vaccaro N, Xi L, et al. Effects of JNJ-38431055, a novel GPR119 receptor agonist, in randomized, double-blind, placebo-controlled studies in subjects with type 2 diabetes. Diabetes Obes Metab (2012) 14:709-16. doi:10.1111/j.1463-1326.2012.01587.x

64. Nunez D, Bush M, Collins D, McMullen S, Gillmor D, Apseloff G, et al. Gut hormone pharmacology of a novel GPR119 agonist (GSK1292263), metformin, and sitagliptin in type 2 diabetes mellitus: results from two randomized studies. PLoS One (2014) 9:e92494. doi:10.1371/journal.pone. 0092494

65. Ritter K, Buning C, Halland N, Pöverlein C, Schwink L. G protein-coupled receptor 119 (GPR119) agonists for the treatment of diabetes: recent progress and prevailing challenges. J Med Chem (2016) 59:3579-92. doi:10.1021/acs. jmedchem.5b01198

66. Cohen L, Esterhazy D, Kim S, Lemetre C, Aguilar R, Gordon E, et al. Commensal bacteria make GPCR ligands that mimic human signalling molecules. Nature (2017) 549:48-53. doi:10.1038/nature23874

67. Park M, Kim S, Ko E, Ahn S, Seo H, Sung M. Gut microbiota-associated bile acid deconjugation accelerates hepatic steatosis in ob/ob mice. J Appl Microbiol (2016) 121:800-10. doi:10.1111/jam.13158

68. Parséus A, Sommer N, Sommer F, Caesar R, Molinaro A, Ståhlman M, et al. Microbiota-induced obesity requires farnesoid X receptor. Gut (2017) 66:429-37. doi:10.1136/gutjnl-2015-310283

Conflict of Interest Statement: The authors declare that the research was conducted in the absence of any commercial or financial relationships that could be construed as a potential conflict of interest.

Copyright (C) $2017 \mathrm{Li}$, Watanabe and Kimura. This is an open-access article distributed under the terms of the Creative Commons Attribution License (CC BY). The use, distribution or reproduction in other forums is permitted, provided the original author(s) or licensor are credited and that the original publication in this journal is cited, in accordance with accepted academic practice. No use, distribution or reproduction is permitted which does not comply with these terms. 